P66 BARRIERS TO ACCESSING PRIMARY CARE FOR SOCIO-
ECONOMICALLY DISADVANTAGED OLDER PEOPLE
LIVING IN RURAL AREAS: A QUALITATIVE STUDY

${ }^{1}$ RT Turley*, ${ }^{1}$ JF Ford, ${ }^{1} \mathrm{TP}$ Porter, ${ }^{1} \mathrm{TS}$ Shakespeare, ${ }^{2} \mathrm{GW}$ Wong, ${ }^{1} \mathrm{AJ}$ Jones, ${ }^{1} \mathrm{NS}$ Steel. ${ }^{1}$ Faculty of medicine and health sciences, University of East Anglia, Norwich, Norfolk; ${ }^{2}$ Nuffield department of primary care health sciences, University of Oxford, Oxford, Oxfordshire

\subsection{6/jech-2017-SSMAbstracts. 167}

Background More healthcare is needed for an ageing population. Within a health care system that has infinite demands and finite resources there is a need to direct those resources available towards those most in need. Previous research has found that older people, those in rural areas, and socio-economically disadvantaged groups are at particular risk of poor access and often have higher health need. We aim to explore the barriers to accessing primary care for socio-economically disadvantaged older people in rural areas.

Methods Semi structured interviews were conducted with fifteen older people over the age of 65 living in a rural area and receiving financial support. Recruitment took place through multiple methods within the community using posters, local radio, hand delivered invitations and invitations dropped in pharmacy bags for home delivery. Participants were purposively sampled for specific characteristics.

Four focus groups were held with rural health professionals from primary care, recruited via the Clinical Research Network.

Thematic analysis was used to identify barriers to primary care access.

Results Multiple barriers were found to impair access to health care of socio-economically disadvantaged older people in rural areas.

From the patient's perspective barriers included attitudes, expectations, experiences and resources. These included engaged telephone lines, availability of appointments, receptionists, transport options, social networks and personal skills. Patients alluded to unwritten rules or a social contract, for example not to bother the doctor in return for additional goodwill when they became unwell. The mismatch between expectations and subsequent experiences led some patients to feel unwelcome or marginalised.

From the health professionals' perspective, barriers included rising demands and expectations, necessitating service reorganisation to overcome significant financial and workforce challenges. Changes included fewer home visits, telephone consultations, triaging calls and appointment system modification.

Conclusion Multiple barriers to accessing primary care exist for this group. As primary care is re-organised to reduce costs, commissioners and practitioners must not lose sight of the perceived social contract and models of care that form the basis of how many older people interact with the service.

Access to services is a key policy area to address inequalities. One-size-fits-all policies may improve access for the whole population leading to increased pressure on the system and counterproductively worse access for marginalised groups. A targeted approach is needed which appreciates and addresses key barriers.

\section{P67 THE RELATIONSHIP BETWEEN SOCIO-ECONOMIC CLASS AND WORK-RELATED MENTAL ILL-HEALTH}

${ }^{1} \mathrm{~L}$ Hussey*, ${ }^{1} \mathrm{~A}$ Money, ${ }^{2} \mathrm{M}$ Gittins, ${ }^{1} \mathrm{R}$ Agius. ${ }^{1}$ Centre for Occupational and Environmental Health, The University of Manchester, Manchester, UK; ${ }^{2}$ Centre for Biostatistics, The University of Manchester, Manchester, UK

\subsection{6/jech-2017-SSMAbstracts. 168}

Background Inequalities in health are often described showing an inverse relationship with social economic group and mortality and morbidity. However, work-related mental ill-health (WRMIH) reported by GPs show highest rates amongst higher socio-economic groups, particularly 'Lower managerial and professional' and 'Intermediate' occupations. Similar results are shown with the self-reported WRMIH collected by the Selfreported Work-related Illness (SWI) survey as part of the Labour Force Survey (LFS). The Health and Occupation Research network (THOR) collects data on WRMIH from GPs, occupational physicians (OPs) and psychiatrists. This study aimed to analyse the psychosocial factors associated with the WRMIH reported in different socio-economic groups.

Methods With each case of WRMIH, participating physicians report information including occupation and the psychological stressor considered to be associated with the condition. Occupational data were coded using the National Statistics SocioEconomic Classification (NS-SEC). A multi-level logistic regression calculated odds ratios (ORs) (adjusted by age and gender) reporting the likelihood of the stressor occurring within each socio-economic group compared to the highest reference group 'Large employers and higher managerial occupations'.

Results Results showed that the socio-economic groups were exposed to significantly different workplace stressors. Cases reported from the highest group were more likely to be associated with workload and workplace change with ORs falling as socio-economic status decreased, e.g. for workload, ORs in the lowest NS-SEC group were 0.51 (GPs), 0.14 (OPs) and 0.16 (psychiatrists). Lower NS-SEC groups were more likely to have problems associated with bullying and interpersonal relationships, e.g. for bullying, ORs in the lowest group were 2.60 (GPs), 1.76 (OPs) and 1.60 (psychiatrists).

Conclusion WRMIH rates may be greatest amongst higher socio-economic groups as they are more likely to have problems associated with a heavy workload and changes in the workplace such as staff shortages and organisational restructure. NS-SEC groups with highest rates such as the 'middling' lower managerial and intermediate occupations may find themselves caught in the middle and experience the "worst of both worlds", i.e. they feel responsibility for the success of the workplace, have demands from those in higher managerial positions, but also have to deal with issues surrounding the 